\title{
Rare Case of Immature Gastric Teratoma
}

\author{
Ivascu M11, Bembea M11, Jurca Claudia1', Moldovan Corina¹, Buicu F² \\ 1 Municipal Clinical Hospital "Dr. Gavril Curteanu" Oradea \\ 2 University of Medicine and Pharmacy of Tirgu-Mures
}

\begin{abstract}
Introduction: Teratomas are rare and complex tumors with components from more than one of the three germ cell layers. Teratomas range from benign, well-differentiated (mature) cystic lesions to those that are solid and malignant (immature). The incidence of all teratomas is estimated at 1:10,000-1:20,000 newborns. Gastric teratomas represent only 1-2\% of all teratomas.

Case presentation: We report a case of gastric teratoma of a 2 month-old boy who presented with abdominal distension. Diagnosis was established by physical examination, ultrasonography and computed tomography. The tumor measured 13/10/5.5 cm and weighted $390 \mathrm{grams}$ and was surgically excised. Histological examination revealed an immature gastric teratoma. We also reviewed existing clinical and genetic data on gastric teratomas. Association of gastric teratomas with other congenital anomalies or tumors is very rare. Reported cases include: Beckwith-Wiedemann syndrome (involved:11p15, IGF2), Hodgkin disease (developed 3 yrs. post-resection) and focal neuroblastoma. Recent theories include extraembryonic cells; also have been hypothesized to originate from pluripotent cells present in the gastric wall.

Conclusion: Gastric teratomas are extremely rare tumors. Complete resection induce a good outcome.
\end{abstract}

Keywords: teratomas, genes, embryonic tumors, gastric tumors

Received: 01 July 2014 / Accepted: 10 August 2014

\section{Introduction}

Teratomas are rare and complex tumors with components from more than one of the three germ cell layers. Teratomas range from benign, well-differentiated (mature) cystic lesions to those that are solid and malignant (immature). The incidence of all teratomas is estimated at 1:100001:20000 newborns. Gastric teratomas represent only 1-2\% of all teratomas.

\section{Case presentation}

This is a case report of an immature gastric teratoma of a two month-old boy, who was presented to our hospital with the main complaint of refusal to feed and abdominal distension.

Present illness history. An abdominal distention was noted by the parents during the first week of life; the patient continued to feed well and there was no history of vomiting, constipation or diarrhea. Two days prior to presentation, the patient developed constipation, refusal to feed and acute worsening of abdominal distention. This was brought to medical attention and he was hospitalized.

Birth history indicated a normal pregnancy, with patient born at full-term, via spontaneous vaginal delivery. His birth weight was 3,000 grams and there was no perinatal distress.

Family history. The patient was the third child of healthy non-consanguineous parents (18 year-old mother, 25 yearold father). He has a 30 month-old sister and a 19 monthold brother, both healthy.

Clinical examination revealed a weight of 4,400 grams (10th percentile) and an altered general appearance, with

Correspondence to: Marius Ivascu

E-mail: ilz875@yahoo.com suffering facies, agitated cry; tachypneea and shallow breaths. We also noted significant abdominal distension with shiny abdominal wall skin, hypogastric and scrotal edema, guarding and inability to palpate abdominal viscera while awake. During sleep, a large, mobile mass extending from the right to the left flank and over the midline was palpated. Rectal exam indicated a normal fecal tone and stool in the rectal vault.

Laboratory tests indicated a normal complete blood count, normal arterial blood gases, normal values for electrolytes, glycemia, liver function tests, alpha-fetoprotein and erythrocyte sediment rate.

Imaging tests:

- Abdominal Ultrasound was non interpretable

- Thoracic and abdominal X-ray indicated an Abdominal distension with gaseous bowel distension in the right flank and inferior abdomen, and opacity in the rest of the abdomen

- Abdominal CT with contrast revealed the presence of an intraperitoneal, supra- and infra-mesocolic, $12 \mathrm{~cm} / 8 \mathrm{~cm}$ mass, which displaced adjacent loops of bowel towards the hypogastrum and the left flank, with caudal and posterior displacement of the spleen and kidneys. The tumor contained large septated cysts, as well as solid iodophile formations and multiple calcifications of the cystic walls and septae.

Treatment consisted in surgical excision of the tumoral mass, $12-13 \mathrm{~cm}$ (Figure 3), which appeared to have an irregular surface with many serous and blood-containing cysts (Figure 4). The tumor arose from the anterior gastric wall (the fundus and gastric body junction) (Figure 5) and displaced the left liver lobe, the spleen and gastric body. The tumor had multiple hard cartilaginous areas alternating with macrocystic areas and it was completely excised by 
circumferential gastrectomy. A $3.5 \mathrm{~cm}$ gastric incision was subsequently closed by transversegastroraphy.

The macroscopic pathologic findings were: multinodular solid and cystic areas; non-continuous tumor capsule (Figure 6). Tumor sections revealed many serous and serosanguineous cysts; the solid areas had a white-yellow color, containing regions of calcification and bone formation and many small cysts with transparent walls; separately a nodular elastic process was identified, $2.1 / 1.5 / 0.9 \mathrm{~cm}$ in dimension, with cysts within solid area.

Microscopic pathologic findings were:

- Solid and cystic areas of proliferative tissues derived from all three germ layers were identified: squamous, intestinal and respiratory epithelium, glandular struc-

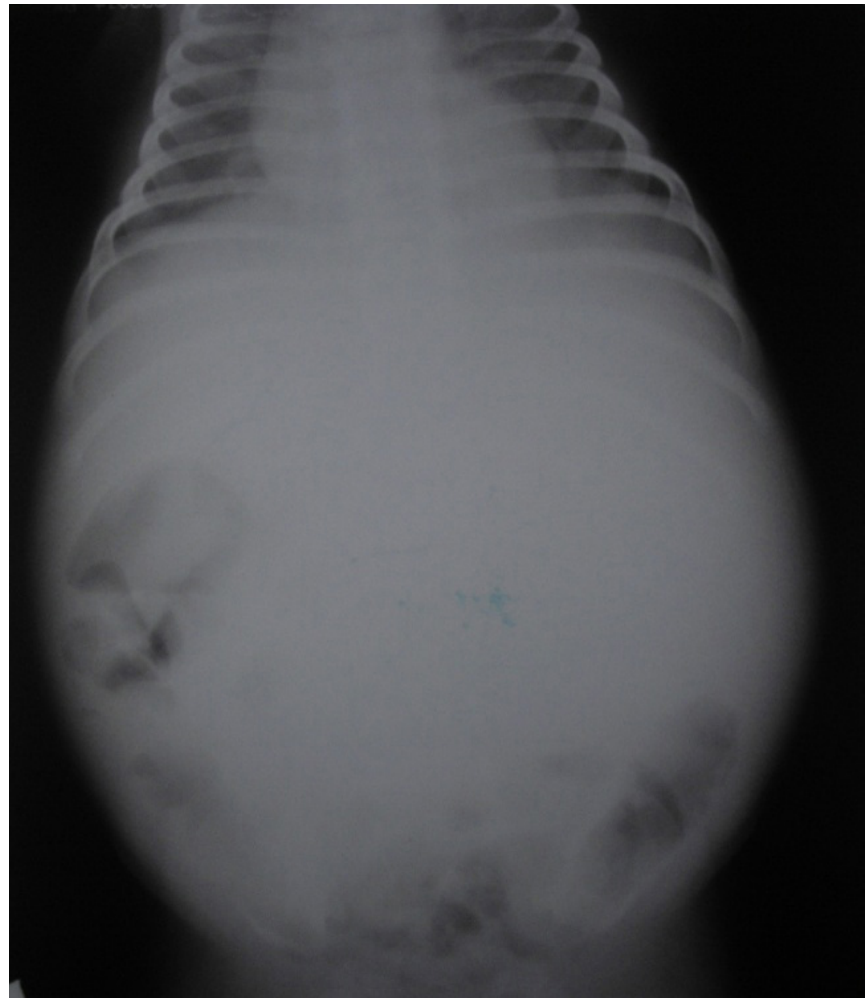

Fig. 1. Thoracic and abdominal X-ray: Abdominal distension with gaseous bowel distension in the right flank and inferior abdomen; opacity in the rest of the abdomen

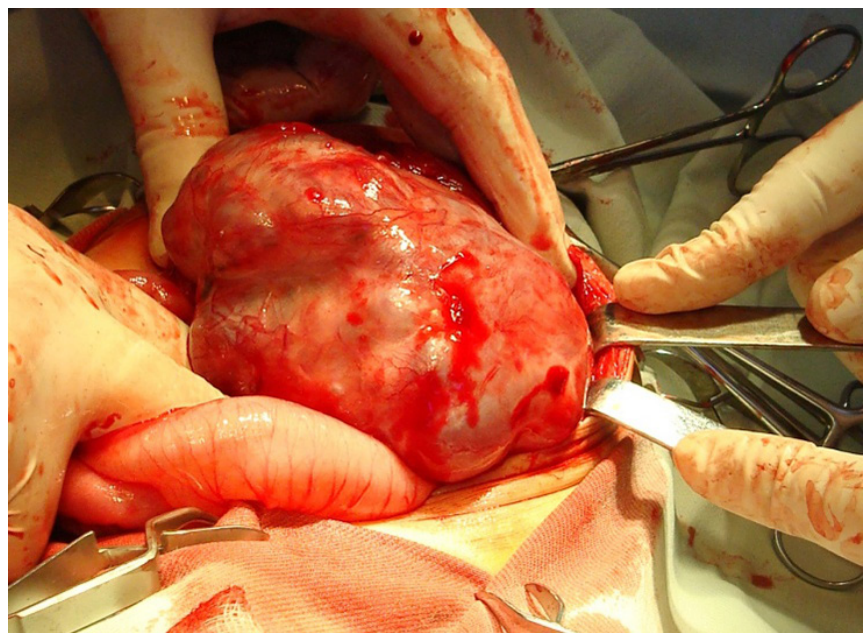

Fig. 3. Intraperitoneal mass, with a $12-13 \mathrm{~cm}$ maximum diameter tures, pilous follicles, muscle, cartilage, bony and adipose tissue, dental and nervous tissue, choroid plexus, mesenchymal tissue and ganglionar nervous cells.

- Neuroectodermal areas were localized as rossettes, neural tubes or glial areas (immature nervous tissue fields/slide).

- Some cysts had papillary projections covered by clear cells or cylindric epithelium. There were small regions containing clear cytoplasma cells forming pseudoglands or trabecullae.

- The specimen contained gastric wall with submucosal hemorrhagic foci covering diverse tissues proliferation (epithelium, cartilage, bone, nervous tissue) similar with the tissue found within the tumor.

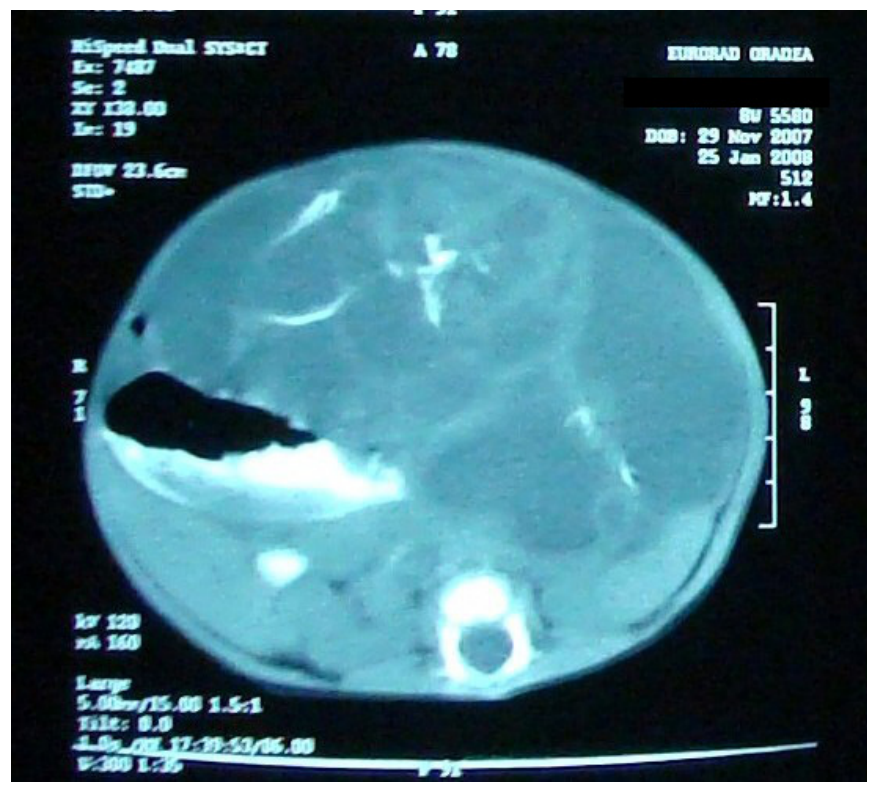

Fig. 2. Abdominal CT with IV Contrast. An intraperitoneal, supra- and infra-mesocolic, $12 \mathrm{~cm} / 8 \mathrm{~cm}$ mass was found. The mass displaced adjacent loops of bowel towards the hypogastrum and the left flank, with caudal and posterior displacement of the spleen and kidneys. The tumor contained large septated cysts, as well as solid iodophile formations and multiple calcifications of the cystic walls and septae.

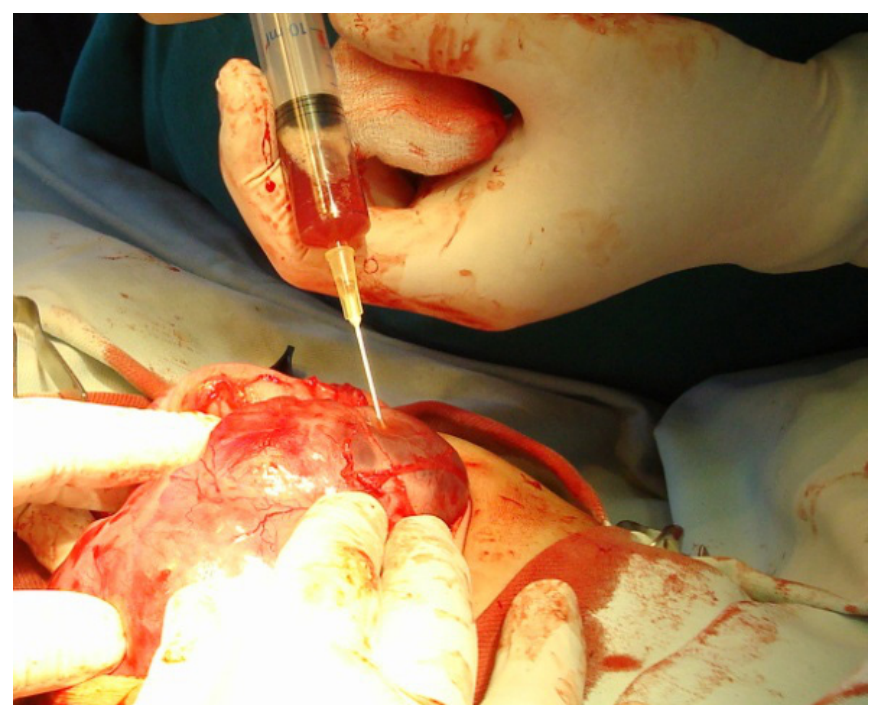

Fig. 4. Serous and blood-containing cysts. 


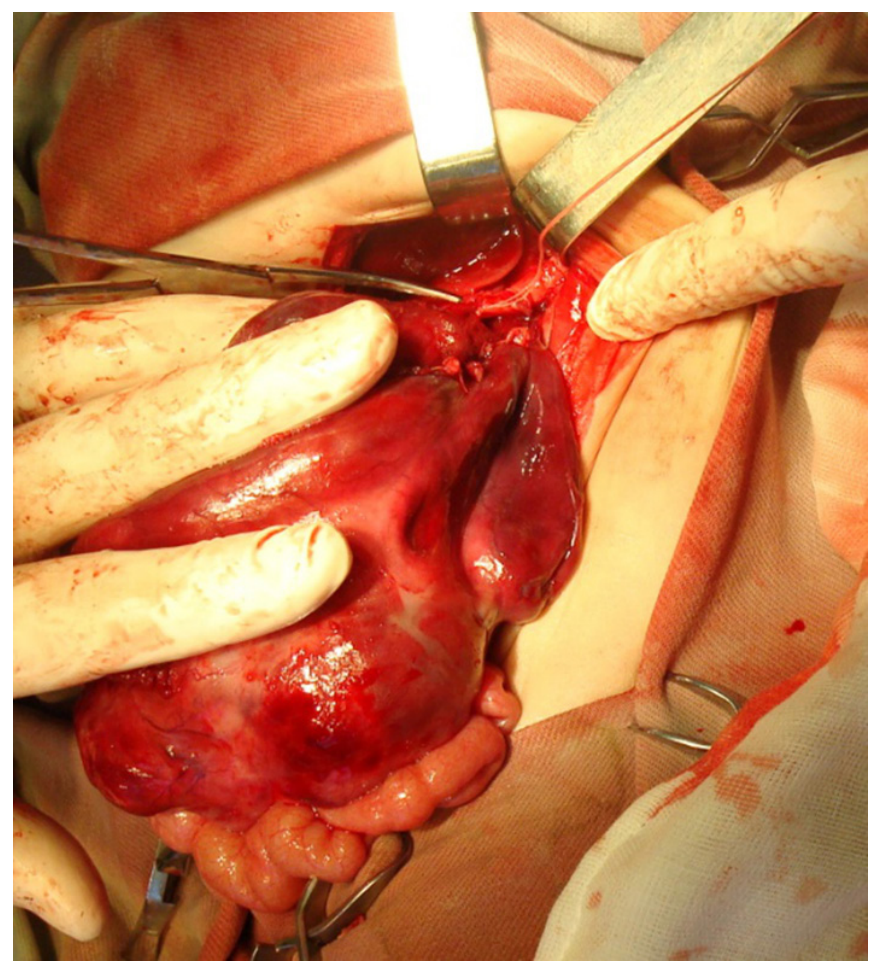

Fig. 5. The tumor adheres to the anterior gastric wall.

- The histopathological specimen was consistent with a 3rd grade, immature gastric teratoma (Figure 7).

Evolution. The patient presented a good post-operative course with normal intestinal transit time and the surgical wound cured per-primam. Clinical follow-up at 1 month, 6 months and 1 year revealed good progress with normal somatic development and normal intestinal transit time.

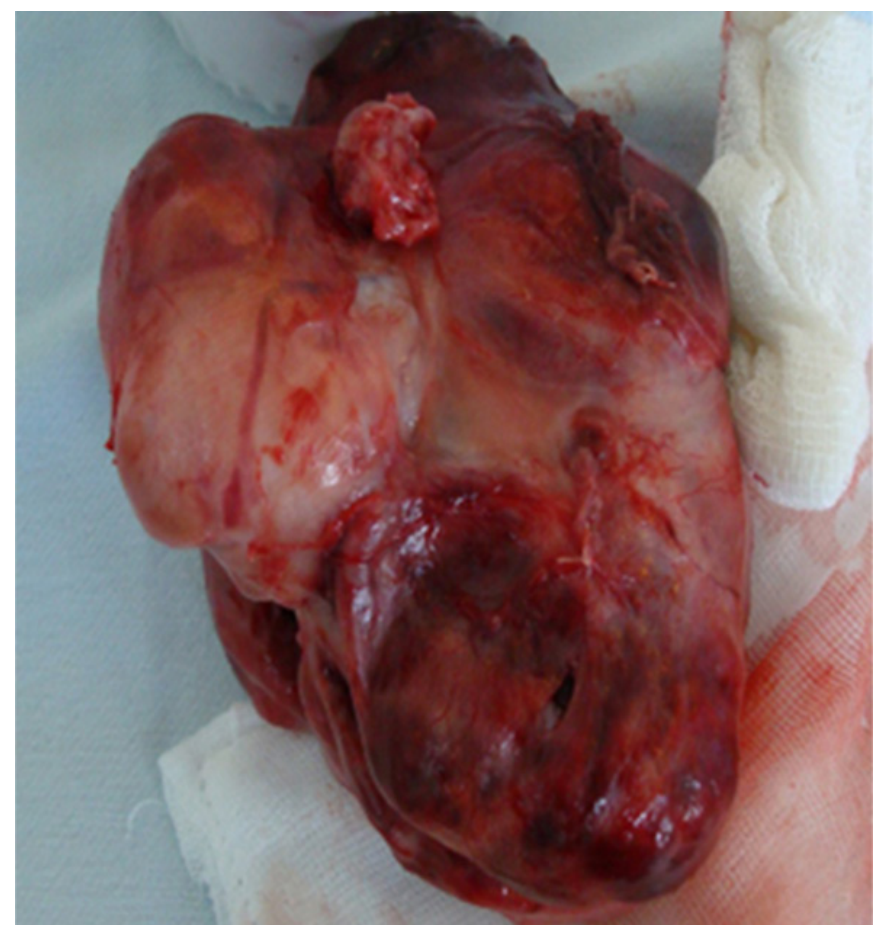

Fig. 6. Large tumor $(13 / 10 / 5.5 \mathrm{~cm}, 390$ grams) adherent to the anterior gastric wall. The arrow shows the excised gastric wall

\section{Discussion}

Teratomas are rare embryonic tumors with components from all three germinal layers. (1). The first case was reported by Eustermann and Sentryin 1922. Teratomas range from benign, well-differentiated (mature) cystic lesions to those that are solid and malignant (immature). Immature teratomas have varying degrees of immature fetal tissues.
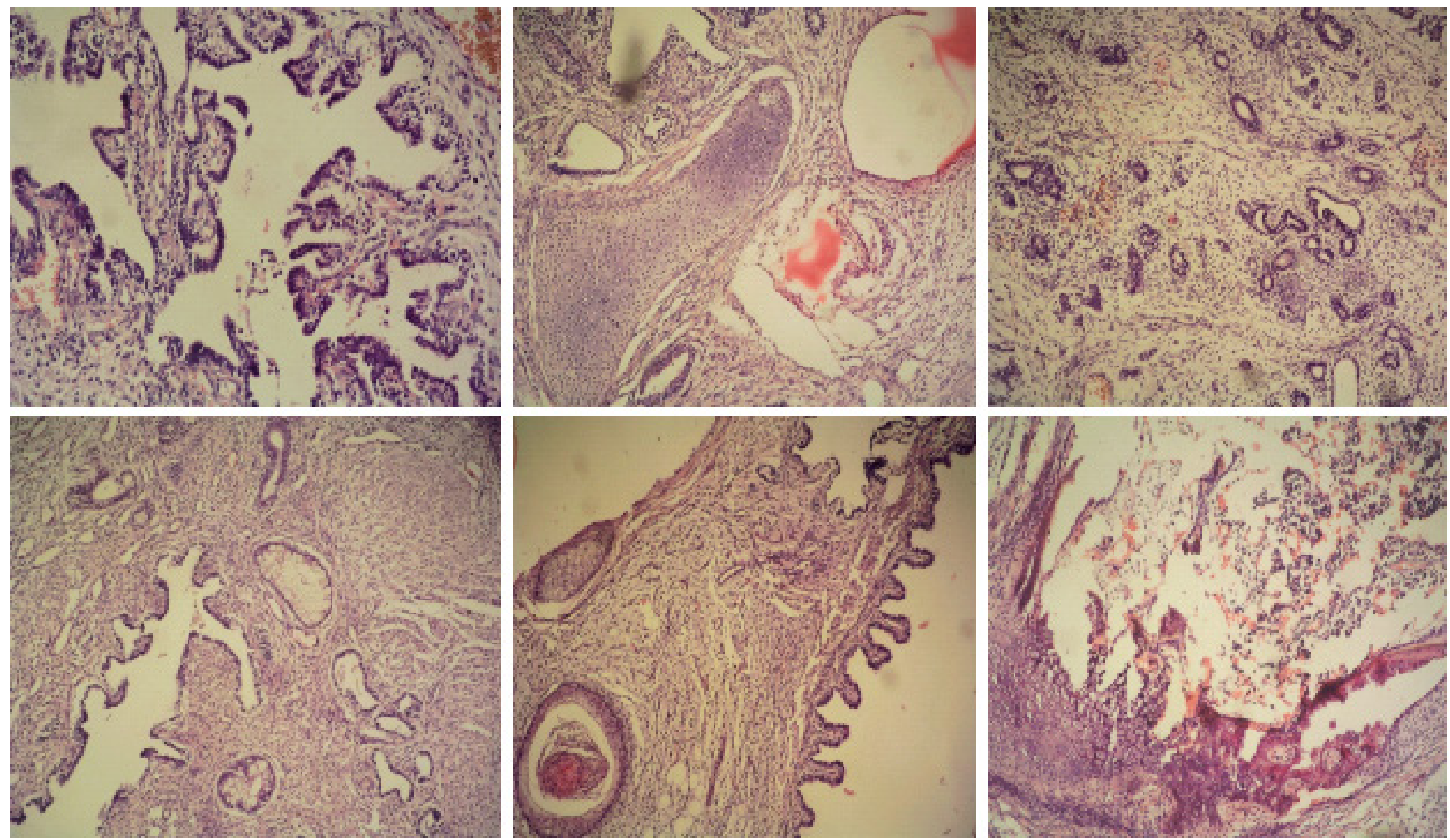

Fig. 7. Histopathological specimen 
The malignant type contains at least one of the malignant germ cell elements. Immature teratomas are graded from 1 to 3 by the amount of immature tissue contents (neural elements) and by the degree of mitotic activity. The incidence of all teratomas is estimated at 1:10,000-1:20,000 newborns.

Gastric teratoma is an extremely rare location: only $1-2 \%$ of all teratomas, i.e. less than 1:1,000,000 newborns. Little more than 100 cases are reported in the literature, out of which only about $10 \%$ are of immature type. More than $90 \%$ of all reported cases are males. Clinical presentation generally occurs within the first 3 months of life but there are few reports of delayed diagnosis in older children and in adolescents [2]. We only found six published cases of gastric teratoma in adults. The most common manifestation of gastric teratomas is abdominal distension, vomiting and an abdominal mass palpated on exam; other described associated signs: gastro-intestinal bleeding (hematemesis or melena), constipation, dyspnea, anemia.

Diagnosis of gastric teratoma is made by US and/or CT. Teratomas have a characteristic heterogenous appearance, including cystic areas with solid components and calcifications. Prenatal diagnosis can be made by fetal US and maternal serum AFP.

Diferential diagnosis include: neuroblastoma, Wilms tumor, hepatoblastoma, liposarcoma; lipoblastoma, rhabdomyosarcoma, retroperitoneal teratoma.

Treatment usually consists in: surgical resection of the entire tumor, or partial gastrectomy may be necessary, as described in our case. Recurrence of tumor is not described after complete resection, and adjuvant therapy (e.g., chemotherapy or radiotherapy) is not needed.

Possible complications and prognosis: Gastric teratomas are usually benign tumors and malignancy is extremely rare (only three cases reported) [1,3,4]. Elevated serum AFP levels may be the only alerting sign of the presence of a malignant teratoma. Correct interpretation of serum AFP levels in infants is dificult because of wide physiologic variation. Acute severe or prolonged bleeding is uncom- mon but a serious complication. However, in our case, post-operative prognosis was excellent.

Genetical aspects: Association of gastric teratomas with other congenital anomalies or tumors is very rare. Reported single cases include: Beckwith-Wiedemann syndrome [5], Hodgkin disease (developed 3 years post-resection) [6] and focal neuroblastoma [7]. No familial cases are reported. Recent theories of teratoma origin include extraembryonic cells other than embryonic stem cells, conjoined and maldeveloped twins, and undetermined cell types. Gastric teratomas also have been hypothesized to originate from pluripotent cells in the gastric wall

\section{Conclusions}

Gastric teratoma is an extremely rare tumor (only 100 reported cases) benign tumor of childhood and the immature type is more rare (not more that 10 cases). This is the first case reported in Romania. The most common physical sign of gastric teratoma is abdominal distension and palpable abdominal mass and abdominal CT is the elective imaging modality of diagnosis. The mature or immature type is defined by histological exam. After complete resection, a good evolution is expected in all cases

\section{References}

1. Gupta DK, Srinivas M, Dave S, Agarwala S, Bajpai M, Mitra DK . Gastric teratoma in children. Pediatr Surg int. 2000;16:329-332.

2. Eastwood GL. Stomach: Anatomy and structural anomalies. In: Textbook of Gastroentroenterology-Yamada. Lippincott Co, 2nd ed. 1995:1312.

3. Balik E, Tunçyürek M, Sayan A, Avanoğlu A, Ulman I, Cetinkurșun S Malignant gastric teratoma in an infant. Z Kinderchir 1990;45:383-385.

4. Bourke CJ, Mackay AJ, Payton D Malignant gastric teratoma: case report. Pediatr Surg Int. 1997;12:192-193.

5. Falik-Borenstein TC, Korenberg JR, Davos I, Platt LD, Gans S, Goodman B, Schreck R, Graham JM Jr. Congenital gastric teratoma in WiedemannBeckwith syndrome, Am J Med Genet. 1991;38(1):52-57.

6. Goetsch, S.J., Hadley, G.P. Hodgkin's disease following successful treatment of gastric teratoma in a neonatal female. Pediatr Pathol Lab Med. 1995;15(3):455-456

7. Hook S, Spicer R, Williams J, Grier D, Lowis S, Foot A, Sergi C. Severe anemia in a 25-day-old infant due to gastric teratoma with focal neuroblastoma. Am J Perinatol. 2003;20(5):233-237. 